\title{
THE SOUL AT INFINITY IN DIMENSION 4
}

\author{
GERARD WALSCHAP \\ (Communicated by Jonathan M. Rosenberg) \\ In memory of Martin Strake
}

\begin{abstract}
It is shown that 4-dimensional open manifolds with nonnegative sectional curvature whose fibers are totally geodesic are metrically rigid. For arbitrary dimension, one also concludes that the curvature is in a sense maximal at the soul.
\end{abstract}

\section{INTRODUCTION}

Let $\left(M^{n}, g\right)$ be an open $n$-manifold with nonnegative sectional curvature $K$ and soul $S$. It is known that there exists a Riemannian submersion $M \rightarrow S$ whenever $n \leq 4$ or the codimension of $S$ is $\leq 2$. In this note, we show that if the fibers of $M \rightarrow S$ are totally geodesic, then $(M, g)$ itself is the result of a Riemannian submersion from a product:

Theorem 1. Let $M^{n}$ be an open n-manifold of nonnegative curvature with soul $S$, where $n \leq 4$ or $\operatorname{codim} S \leq 2$. If the fibers of $M \rightarrow S$ are totally geodesic, then there exists a Riemannian submersion $N \times P^{k} \rightarrow M$. Here $N$ is compact, $P^{k}$ is diffeomorphic to $\mathbb{R}^{k}$, both factors have metrics of nonnegative curvature, and $N \times P^{k}$ has the product metric.

Roughly speaking, $N$ is the soul of the Hausdorff $\operatorname{limit}_{t \rightarrow \infty}(M, \gamma(t))$, where $\gamma$ is a ray from $S$. The proof of Theorem 1 relies heavily on the existence of a Riemannian submersion $M \rightarrow S$. It is not hard to see, however, that one always has an infinitesimal submersion along rays from $S$. In fact, we have the following weak version of O'Neill's formula for curvature:

Theorem 2. Let $M^{n}$ be an open n-manifold with nonnegative curvature $K$ and soul $S$. Let $\gamma:[0, \infty) \rightarrow M$ be a ray starting from $S$, and $P_{t}$ be the parallel translate along $\left.\gamma\right|_{[0, t]}$ of some 2-plane $P_{0} \subset T_{\gamma(0)} S$. Then the function $t \mapsto K_{P_{t}}$ is nonincreasing on $[0, \infty)$.

Received by the editors February 9, 1990 and, in revised form, March 21, 1990.

1980 Mathematics Subject Classification (1985 Revision). Primary 53C20.

Key words and phrases. Riemannian submersion, soul.

The author's research was supported in part by a grant from the NSF. 


\section{Proof of Theorem 1}

The reader is referred to [2] for the general facts concerning nonnegative curvature, to [6,7] for the $\operatorname{codim} S=2$ case, and to [1] or [4] for Riemannian submersions. We shall discuss the two cases (namely $\operatorname{dim} M \leq 4$, and $\operatorname{dim} M>$ 4 but $\operatorname{codim} S \leq 2$ ) simultaneously. Notice first that $M$ may be assumed to be simply connected with soul of codimension 2 . For if $\operatorname{dim} M \leq 4$, then the universal covering map $\tilde{M} \rightarrow M$ is a Riemannian submersion, and since these submersions are preserved under composition, we need only establish the theorem for $\tilde{M}$. But the soul $\tilde{S}$ of $\tilde{M}$ must have codimension 1 or 2 , and in the first case, $\tilde{M}$ splits isometrically as $\tilde{S} \times \mathbb{R}$. Next, suppose $\operatorname{dim} M>4$ and $\operatorname{codim} S \leq 2$. Consider once again the universal covering $\rho: \tilde{M} \rightarrow M$, and let $\tilde{S}=\rho^{-1}(S)$. By [2] $\tilde{S}=S_{0} \times \mathbb{R}^{k}$, with $S_{0}$ compact, simply connected, and since $\tilde{S}$ is totally convex, there is a corresponding splitting $\tilde{M}=M_{0} \times \mathbb{R}^{k}$. Then $S_{0}$ is a soul of $M_{0}$, of codimension $=\operatorname{codim} S$, which by the above may be assumed to equal 2, thereby establishing the claim.

Finally, we may assume that the normal bundle $\nu(S)$ of $S$ in $M$ is not flat, for otherwise, $M=S \times P^{2}$ isometrically. It follows that every geodesic orthogonal to $S$ is a ray, and the exponential map $\exp _{\nu}: \nu(S) \rightarrow M$ is a diffeomorphism, cf. [6, 7]. Let

$$
S(r)=\{q \in M / d(q, S)=r\}
$$

denote the distance sphere of radius $r$ around $S$ with its metric $g_{r}$ induced from $M$.

2.1. Lemma. $\left(S(r), g_{r}\right)$ converges as $r \rightarrow \infty$ to $\left(N, g_{\infty}\right)$, where $N$ is diffeomorphic to the unit sphere bundle $\nu^{1}(S)$, and $g_{\infty}$ is a $C^{\infty}$ metric of nonnegative curvature.

Proof. Given $u \in \nu(S)$, let $\Psi_{u}$ denote the canonical vector space isomorphism between the fiber through $u$ and its tangent space at $u$. One has the polar coordinate vector field $\partial_{\theta}$ on $M \backslash S$ given by $\partial_{\left.\theta\right|_{\text {exp } u}}=\exp _{*} \Psi_{u} J_{u}$, where $J$ is the complex structure on $\nu(S)$-recall that $S$ is simply connected. Since the fibers of $M \rightarrow S$ are totally geodesic, $\partial_{\theta}$ is a Killing field on $M$, cf. [6, Lemma 1.7]. In particular, $G:=\left|\partial_{\theta}\right|$ is a function which depends only on the distance from the soul. Moreover, $G:[0, \infty) \rightarrow \mathbb{R}$ is a concave, increasing, bounded function [6]. Set $\alpha:=\lim _{r \rightarrow \infty} G(r)<\infty$. Notice that $\left(S(r), g_{r}\right)$, being convex, has nonnegative curvature by the Gauss equations, and the projection $S(r) \rightarrow S$ is a Riemannian submersion with iotally geodesic fiber $S^{1}$ generated by $\partial_{\theta}$. Define $\varphi_{r}: \nu^{1}(S) \rightarrow S(r)$ by $\varphi_{r}(v)=\exp (r v)$. Then $\varphi_{r}^{*} g_{r}$ is just the standard connection metric with $\left|\partial_{\theta}\right|$ rescaled by $G(r)$. Since $G(r) \rightarrow \alpha<\infty$, the lemma follows.

2.2. Remark. $\left(N, g_{\infty}\right)$ can be viewed as the soul at infinity in the following sense: let $\gamma:[0, \infty) \rightarrow M$ be a ray from $S$. Then the Hausdorff limit $\lim _{t \rightarrow \infty}^{H}(M, \gamma(t))=(X, 0)$, where $X$ is isometric to $\left(N, g_{\infty}\right) \times \mathbb{R}$. 
Resuming the proof of Theorem 1, recall that since $\nu(S)$ is a complex line bundle, $N \approx \nu^{1}(S)$ is just the corresponding principal $S^{1}$-bundle, and $M$ is diffeomorphic to $\left(N \times \mathbb{R}^{2}\right) / S^{1}$. Endow $\mathbb{R}^{2}$ with the metric $\check{g}=d r^{2}+$ $f^{2}(r) d \theta^{2}$, where $f^{2}(r)=\alpha^{2} G^{2}(r) /\left(\alpha^{2}-G^{2}(r)\right)$. Then the diagonal action of $S^{1}$ on $\left(N, g_{\infty}\right) \times\left(\mathbb{R}^{2}, \check{g}\right)$ is by isometries. We claim that $M$ is isometric to $\left(N \times \mathbb{R}^{2}\right) / S^{1}$. To see this, notice that $\left(N \times \mathbb{R}^{2}\right) / S^{1}$ is topologically $[0, \infty) \times$ $N / \simeq$, where the fibers of $N$ are collapsed at 0 , and the metric has the form $d r^{2}+d \sigma_{r}^{2}$, where $d \sigma_{r}^{2}$ is a metric on $N$ obtained by rescaling in the fiber direction only. It thus suffices to check that the respective Killing fields of $M$ and $\left(N \times \mathbb{R}^{2}\right) / S^{1}$ have the same norm. Now, if $\rho$ denotes the Riemannian submersion $\rho: N \times \mathbb{R}^{2} \rightarrow\left(N \times \mathbb{R}^{2}\right) / S^{1}$, then pointwise, the Killing field on the quotient is $\rho_{*}(0, \partial / \partial \theta)$, where $\partial / \partial \theta$ is the polar coordinate vector field on $\mathbb{R}^{2}$. Since the vertical space of $\rho_{*}$ is spanned by $\left(\partial_{\theta},-\partial / \partial \theta\right)$, one computes, for the horizontal component $(0, \partial / \partial \theta)^{h}$ :

$$
\begin{aligned}
\left(0, \frac{\partial}{\partial \theta}\right)^{h} & =\left(0, \frac{\partial}{\partial \theta}\right)-\left\langle\left(0, \frac{\partial}{\partial \theta}\right),\left(\partial_{\theta},-\frac{\partial}{\partial \theta}\right)\right\rangle \frac{1}{\alpha^{2}+f^{2}}\left(\partial_{\theta},-\frac{\partial}{\partial \theta}\right) \\
& =\frac{1}{\alpha^{2}+f^{2}}\left(f^{2} \partial_{\theta}, \alpha^{2} \frac{\partial}{\partial \theta}\right) .
\end{aligned}
$$

Thus

$$
\left|\rho_{*}\left(0, \frac{\partial}{\partial \theta}\right)\right|=\left|\left(0, \frac{\partial}{\partial \theta}\right)^{h}\right|=\frac{\alpha f}{\left(\alpha^{2}+f^{2}\right)^{1 / 2}}=G \text {. }
$$

2.3. Remark. It is sometimes possible to obtain $M$ from a Riemannian submersion even when the fibers of $M \rightarrow S$ are not totally geodesic. One such example is $M=S^{2} \times \mathbb{R}^{2}$. Here, the metric on $M$ is not a product metric, but comes from a submersion $S^{2} \times \mathbb{R}^{3} \rightarrow M$. On the other hand, consider $S^{3} \times \mathbb{R}^{2}$ with the standard metric, and let $S^{1}$ act diagonally by isometries, on $S^{3}$ via the Hopf fibration, and on $\mathbb{R}^{2}$ by rotations. Then $M:=\left(S^{3} \times \mathbb{R}^{2}\right) / S^{1} \rightarrow S=$ $S^{3} / S^{1} \approx S^{2}$ has totally geodesic fibers. Nevertheless, there exists a deformation $g_{\varepsilon}$ of the metric $g$ on $M$ (with nonnegative sectional curvature), such that $\left(M, g_{\varepsilon}\right)$ is not isometrically a quotient $\left(S^{3} \times \mathbb{R}^{2}\right) / S^{1}$ for any metrics on $S^{3}$ and $\mathbb{R}^{2}$. Of course, the fibers of $M \rightarrow S$ are no longer totally geodesic. For details of both examples, see [6].

\section{Proof of Theorem 2}

We briefly recall some well-known facts about convex sets in a Riemannian manifold $M$ of nonnegative sectional curvature. The reader should consult $[2,5]$ for proofs and further details. If $C \subset M$ is convex with nonempty boundary $\partial C$, and $\rho: C \rightarrow \mathbb{R}, \rho(p):=d(p, \partial C)$, denotes the distance function to the boundary, then $C^{a}:=\{q \in C / \rho(q) \geq a\}$ is again convex. Moreover, if $C^{a} \neq \varnothing$, there exists a deformation retraction $\varphi: C \times[0, a] \rightarrow C^{a}$ which is distance nonincreasing. In general, the curves $t \mapsto \varphi(p, t)$, which 
can be regarded as integral curves of a generalized gradient $\nabla \rho$, need not be smooth. It is, however, easy to check that by the very construction of $\varphi$ (see [5]) given $q \in C^{a}$, and a minimal geodesic $\gamma$ from $q$ to $\partial C,-\gamma$ is, up to reparametrization, an integral curve of $\nabla \rho$.

3.1. Lemma. Let $S$ be a soul of $M$, and let $\gamma_{i}$ be rays with $\gamma_{i}(0) \in S, i=$ 1,2 . Then the function $t \mapsto d\left(\gamma_{1}(t), \gamma_{2}(t)\right)$ is nondecreasing.

Proof. Performing the first step in the soul construction starting at $\gamma_{1}(0)$, we have, with the notation of [2], a family $\left\{C_{t}\right\}_{t \geq 0}$ of totally convex sets, with

$$
S \subset C_{0}=\left\{q \in C_{t} / d\left(q, \partial C_{t}\right)=t\right\} .
$$

Fix any $t>0$, and let $\rho$ denote the distance function from $\partial C_{t}$. By definition, any ray from $\gamma_{1}(0)$ is a minimal connection to $\partial C_{t}$. But if $u \in T_{\gamma_{1}(0)} M$ denotes the parallel translate of $\dot{\gamma}_{2}(0)$ along some geodesic from $\gamma_{2}(0)$ to $\gamma_{1}(0)$, then $t \mapsto \exp (t u)$ is a ray (cf. [6]). It follows from [2, Theorem 1.10] that $\gamma_{2}$ itself is a minimal connection to $\partial C_{t}$. Thus both $\gamma_{1}$ and $\gamma_{2}$ are, up to negative reparametrization, integral curves of $\nabla \rho$. This establishes the lemma.

To complete the proof of Theorem 2, let $x, y$ be an orthonormal basis of $P_{0}, X, Y$ the parallel vector fields along $\gamma$ with $X(0)=x, Y(0)=y$. For $t \geq 0, r>0$, consider the circles $C_{r, t}:[0,2 \pi] \rightarrow M$,

$$
C_{r, t}(\theta)=\exp _{\gamma(t)} r(\cos \theta \cdot X(t)+\sin \theta \cdot Y(t)) .
$$

By [2, Theorem 1.10], the minimal geodesic from $C_{r, 0}(\theta)$ to $C_{r, t}(\theta)$ is a ray: indeed its initial tangent vector is the parallel translate of $\dot{\gamma}(0)$ along $s \mapsto \exp _{\gamma(0)} s(\cos \theta \cdot x+\sin \theta \cdot y)$. By 3.1, we have

$$
d\left(C_{r, t}(\theta), C_{r, t}\left(\theta^{\prime}\right)\right) \leq d\left(C_{r, t^{\prime}}(\theta), C_{r, t^{\prime}}\left(\theta^{\prime}\right)\right)
$$

for all $\theta, \theta^{\prime}, t \leq t^{\prime}$. Thus the length of $C_{r, t}$ is not bigger than the length of $C_{r, t^{\prime}}$, if $t \leq t^{\prime}$. The theorem now follows from a well-known formula relating the length of $C_{r, t}$ as $r \rightarrow 0$ with the sectional curvature of $P_{t}$, see for example [3, p. 124].

3.2. Question. Theorem 2 says grosso modo that the curvature is maximal at the soul. Notice that $t \mapsto K_{P_{t}}$ need not be strictly decreasing, as in the case when $M=S \times P^{k}$ isometrically. More generally, suppose $R(x, y) \dot{\gamma}(0)=0$. When the projection $M \rightarrow S$ is a Riemannian submersion, it is not hard to check that $K_{P_{t}}$ is constant. Is this still true in general?

\section{REFERENCES}

1. A. Besse, Einstein manifolds, Ergeb. Math. Grenzgeb. (3), Bd. 10, Springer-Verlag, Berlin and Heidelberg, 1987.

2. J. Cheeger and D. Gromoll, On the structure of complete manifolds of nonnegative curvature, Ann. of Math. 96 (1972), 413-443. 
3. S. Gallot, D. Hulin, and J. Lafontaine, Riemannian geometry, Universitext, Springer-Verlag, Berlin and Heidelberg, 1987.

4. B. O'Neill, The fundamental equations of a submersion, Michigan Math. J. 13 (1966), 459469.

5. V. A. Sharafutdinov, The Pogorelov-Klingenberg theorem for manifolds homeomorphic to $\mathbb{R}^{n}$, Sibirsk. Mat. Z 18 (1977), 915-925.

6. G. Walschap, Nonnegatively curved manifolds with souls of codimension 2, J. Differential Geom. 27 (1988), 525-537.

7. _ A splitting theorem for 4-dimensional manifolds of nonnegative curvature, Proc. Amer. Math. Soc. 104 (1988), 265-267.

Department of Mathematics, University of California, Los ANgeles, California 90024 